\title{
Effects of Coordinately Unsaturated Cobalt Complexes on Radical Polymerization of Vinyl Monomers
}

\author{
V. A. BHANU and K. KISHORE, * Department of Inorganic and Physical \\ Chemistry, Indian Institute of Science, Bangalore-560 012, India
}

\begin{abstract}
Synopsis
The tetrahedral cobalt (II) complexes A-D are found to possess an unusual coordinately unsaturated nature. These additives significantly inhibit the thermal polymerization of acrylamide and further, they impart significant induction period during AIBN initiated polymerization of MMA and acrylamide. Some evidence has been obtained to show that the additives' effect plausibly results because of the reaction of the coordinately unsaturated cobalt complexes with radicals present in the system.
\end{abstract}

\section{INTRODUCTION}

Transition metal complexes are being currently studied as additives ${ }^{1-3}$ rather than as initiators ${ }^{4}$ in radical polymerization of vinyl monomers. Some of the fascinating developments in this interdisciplinary field are: catalytic chain transfer, ${ }^{5,6}$ control of the architecture of copolymers, ${ }^{7}$ etc. These stem from the possibility of the transition metal ion interacting with "suitable" ligands present in the polymerization medium.

In the case of cobalt additives, both square planar and tetrahedral complexes contain coordinately unsaturated cobalt ions. Understandably therefore, the interaction of square planar cobalt (II) complexes with suitable ligands has been studied. ${ }^{8}$ Further, the interaction of these complexes with alkyl radicals is an active topic of contemporary research owing to its implications in the fields of organometallic ${ }^{9}$ and biomedical ${ }^{10}$ chemistry. Nevertheless, in contrast to the square planar complexes, the tetrahedral cobalt complexes generally contain sterically bulky ligands which forbid them from coordinating with extra ligands. Consequently, the parallel chemistry of tetrahedral cobalt complexes is not yet available. However, in the case of complex A (Scheme 1) it has been observed $^{11}$ that despite being a tetrahedral complex, it can easily accommodate two extra ligands, as reflected by the significant change in the near-IR spectrum of the complex in benzene. This transformation of tetrahedral to octahedral geometry demonstrates the unusual flexibility of this complex to coordinate with other suitable ligands. A similar geometrical transformation of the other tetrahedral cobalt complexes B, C, and D (Scheme 1) has been confirmed by us and a representative example is presented in Figure 1.

Due to this interesting behavior, we anticipate complexes A-D to bring about significant changes in the radical polymerization reactions. We have therefore studied the thermal and AIBN (2,2'-azobisisobutyronitrile) initiated polymer-

* To whom correspondence should be addressed.

Journal of Polymer Science: Part A: Polymer Chemistry, Vol. 28, 3617-3624 (1990) (C) 1990 John Wiley \& Sons, Inc.

CCC 0887-624X/90/133617-08\$04.00 


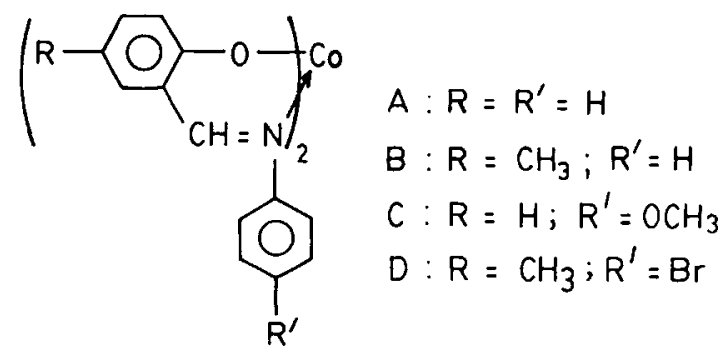

Scheme 1. Structure of cobalt complexes.

ization of acrylamide in the presence of the above additives by differential scanning calorimetry (DSC). Also, polymerization of styrene and MMA (methyl methacrylate) has been studied by dilatometry in the presence of these additives.

\section{EXPERIMENTAL}

Acrylamide (J. T. Baker) was twice recrystallized from acetone ( $\mathrm{mp} 84^{\circ} \mathrm{C}$ ). AIBN (Fluka) was twice recrystallized from methanol. Styrene and MMA monomers were purified by removing the inhibitor by alkali treatment followed by distillation under reduced pressure. The cobalt complexes were prepared as reported elsewhere. ${ }^{12}$

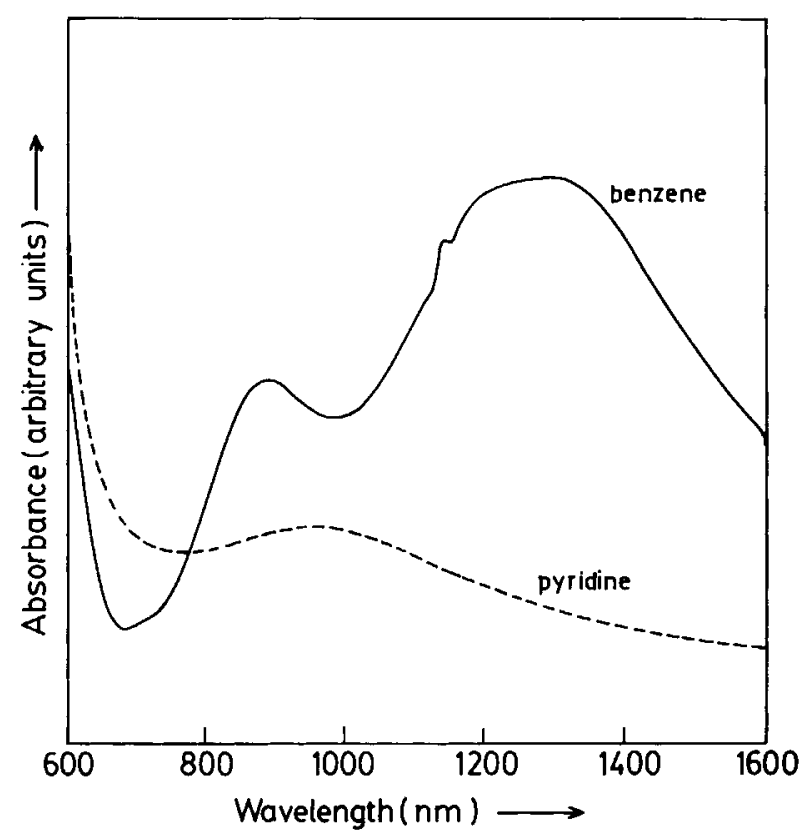

Fig. 1. Near-IR spectra of complex B in different solvents. 
The near-IR spectra of the complexes were recorded on a Hitachi U-3400 spectrophotometer. The polymerization of acrylamide was studied using a Dupont 990 DSC, under nitrogen atmosphere, and conditions mentioned in Figures 2 and 3 . The dilatometric studies were carried out using a dilatometer of 4.5 $\mathrm{mL}$ capacity which was calibrated for conversions up to $5 \%$ using various combinations of monomer and AIBN concentrations in conjunction with independent gravimetric estimation of polymer precipitated from methanol. The following calibration equations for the rate of polymerization $\left(R_{p}\right)$ were obtained from the slope $(S)$ of the plot of extent of conversion (measured as decrease in solution level in the dilatometer) against time.

For styrene

$$
R_{p}=7.01 \times 10^{-3} \times S
$$

For $M M A$

$$
R_{p}=5.85 \times 10^{-3} \times S
$$

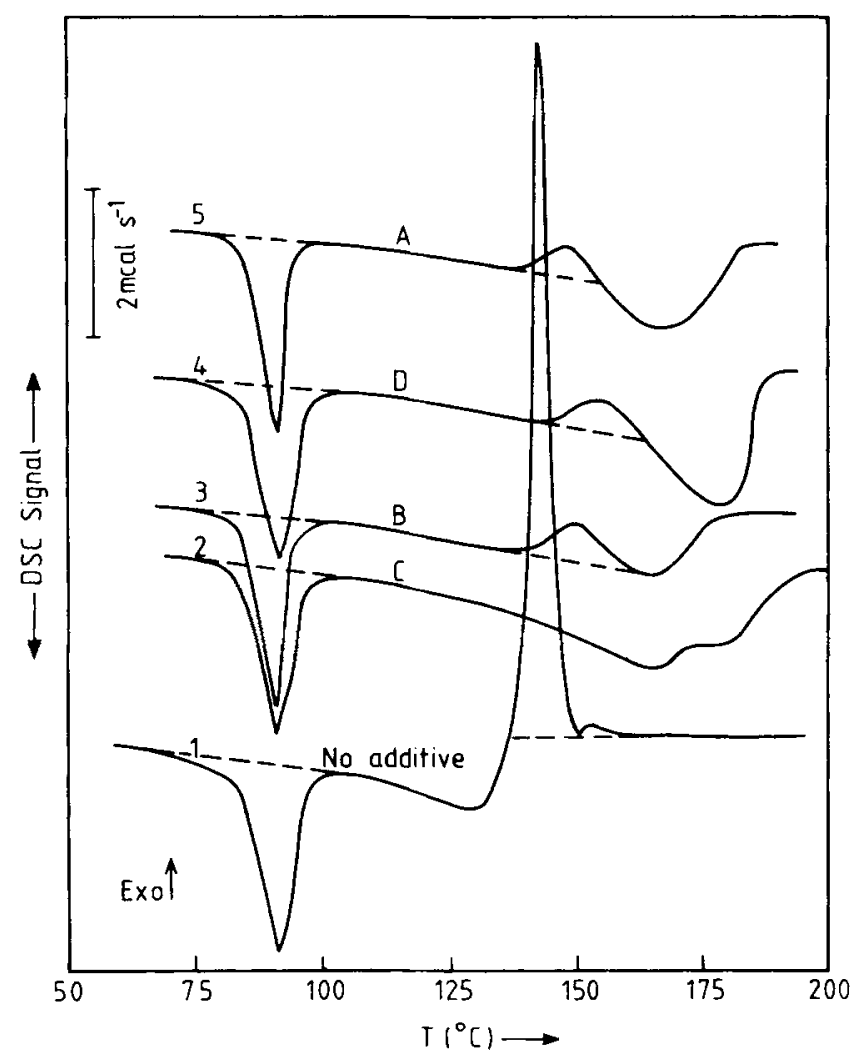

Fig. 2. DSC thermograms for thermal polymerization of acrylamide. Conditions: nitrogen atmosphere; heating rate $=10^{\circ} \mathrm{C} / \mathrm{min}$; chart speed: $0.5 \mathrm{~min} / \mathrm{cm}$; sensitivity: $20 \mathrm{mV} / \mathrm{cm}$. Sample weight: 1 , (acrylamide: $4 \mathrm{mg}$ ); 2-4, (acrylamide: $4 \mathrm{mg}+$ additive: $2 \mathrm{mg}$ ). 


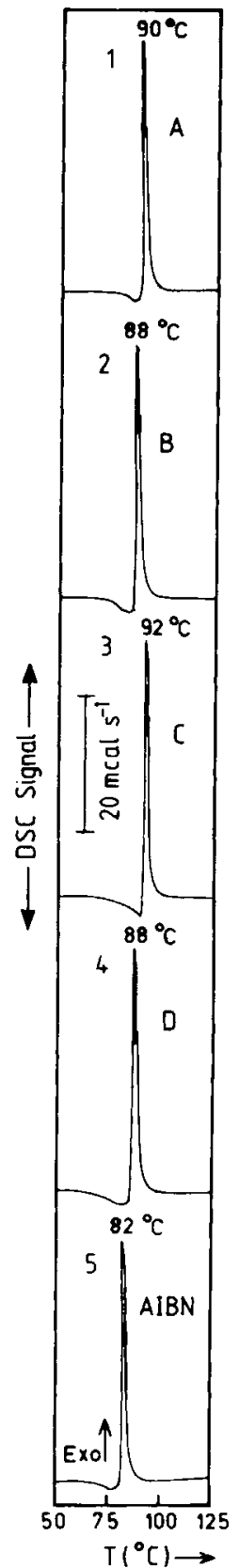

Fig. 3. DSC thermograms for AIBN initiated polymerization of acrylamide. Conditions: nitrogen atmosphere; heating rate: $10^{\circ} \mathrm{C} / \mathrm{min}$; chart speed: $0.5 \mathrm{~min} / \mathrm{cm}$; sensitivity: $200 \mathrm{mV} / \mathrm{cm}$. Sample weight: 1-4, (acrylamide: $4 \mathrm{mg}+$ AIBN: $2 \mathrm{mg}+$ additive: $2 \mathrm{mg}$ ); 5, (acrylamide: $4 \mathrm{mg}+$ AIBN: $2 \mathrm{mg}$ ).

\section{RESULTS AND DISCUSSION}

The effect of additives on the thermal and AIBN induced polymerization of acrylamide is shown in Figures 2 and 3, respectively. It is well known ${ }^{13}$ that 
during thermal polymerization, acrylamide first melts and then polymerizes exothermically (thermogram 1, Fig. 2 ). In the presence of additives A-D, the polymerization exotherm is completely suppressed (thermograms $2-5$, Fig. 2) and the polymerization is completely inhibited in the presence of additive $C$. On comparing the area under the exotherm in absence and presence of additives, it is observed that additives allow conversion of the order of only $10 \%$ (Table I). Also, the peak temperature $\left(T_{p}\right)$ of the polymerization exotherm shifts to higher temperatures in the presence of additives (Table I).

For AIBN initiated polymerization, acrylamide polymerizes soon after it melts (thermogram 5, Fig. 3); the fusion endotherm is not as distinct as in the case of thermal polymerization. In the presence of additives, the overall pattern is the same except that $T_{p}$ is higher in all the cases (thermograms $1-4$, Fig. 3 and Table I). Further, unlike in the case of thermal polymerization, the area under the polymerization exotherm as well as the maximum rate of heat release (measured as the ordinate value in the thermograms at $T_{p}$ ) remains unaffected in the presence of additives (Table I).

These results show that the additives A-D strongly inhibit the thermal polymerization of acrylamide. As regards the AIBN induced polymerization, though the polymerization may be taking place in the melt and/or the solid state (since the fusion endotherm is not distinctly separated from the polymerization exotherm), an inhibitory effect of the additives is evident. This has been further substantiated by independent solution polymerization experiments. The thermal polymerization of acrylamide in benzene at reflux in nitrogen atmosphere does not yield a precipitate of the polymer even after an hour of refluxing. Copious amounts of polymer however, are found to precipitate within $0.5 \mathrm{~h}$ under similar conditions but in the absence of the additives. Similarly, in case of AIBN induced polymerization, no polymer was found to precipitate in presence of the additives after refluxing for an additional $5 \mathrm{~h}$.

TABLE I

DSC Data for Acrylamide Polymerization

\begin{tabular}{|c|c|c|c|c|c|}
\hline \multirow[b]{2}{*}{ Parameters } & \multicolumn{5}{|c|}{ Additives } \\
\hline & None & A & B & $\mathrm{C}$ & $\mathrm{D}$ \\
\hline \multicolumn{6}{|l|}{ Thermal polymerization ${ }^{*}$} \\
\hline $\begin{array}{l}\text { Monomer/additive } \\
\text { (mol ratio) }\end{array}$ & \multicolumn{5}{|c|}{ Monomer/additive } \\
\hline$T_{p}\left({ }^{\circ} \mathrm{C}\right)$ & $135 \pm 2$ & $142 \pm 2$ & $147 \pm 2$ & - & $155 \pm 2$ \\
\hline Relative polymerization $(\%)$ & 100 & 8.5 & 8.8 & - & 8.0 \\
\hline \multicolumn{6}{|l|}{ AIBN induced polymerization ${ }^{\mathrm{a}, \mathrm{b}}$} \\
\hline \multicolumn{6}{|l|}{ Monomer/additive } \\
\hline (mol ratio) & - & 13.66 & 12.73 & 14.36 & 15.56 \\
\hline$T_{p}\left({ }^{\circ} \mathrm{C}\right)$ & $82 \pm 2$ & $90 \pm 2$ & $88 \pm 2$ & $92 \pm 2$ & $88 \pm 2$ \\
\hline $\begin{array}{l}\text { Rate of heat release at } T_{p} \\
\quad\left(\text { mcal s}^{-1}\right)\end{array}$ & $102 \pm 2$ & $102 \pm 2$ & $102 \pm 2$ & $102 \pm 2$ & $102 \pm 2$ \\
\hline
\end{tabular}

acrylamide] $=5.6 \times 10^{-5} \mathrm{~mol}$. Other conditions: nitrogen atmosphere; sensitivity $=20 \mathrm{mV}$

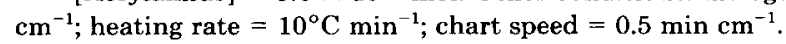

${ }^{\mathrm{b}}$ [AIBN] $=1.22 \times 10^{-5} \mathrm{~mol}$; sensitivity $=200 \mathrm{mV} \mathrm{cm}^{-1}$. 


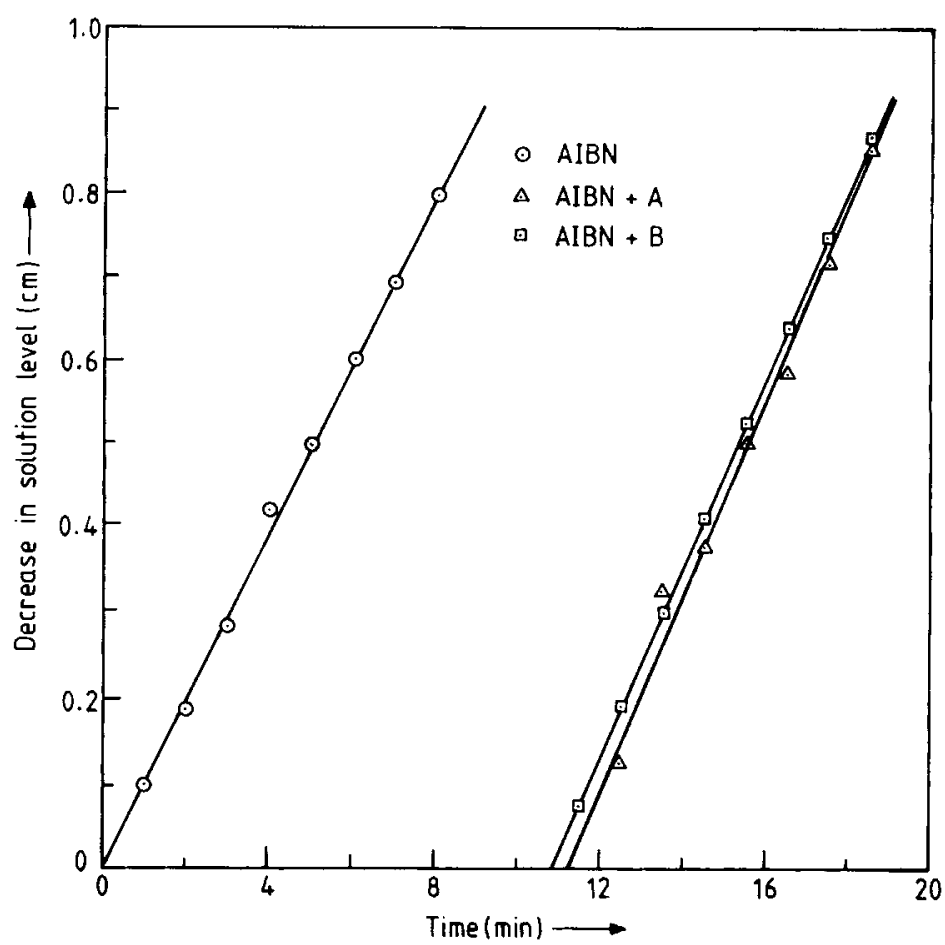

Fig. 4. Dilatometric measurement of time against decrease in solution level in MMA polymerization at $65^{\circ} \mathrm{C}$. $[\mathrm{AIBN}]=0.013 \mathrm{~mol} / \mathrm{L} ;[\mathrm{A}]=[\mathrm{B}]=0.009 \mathrm{~mol} / \mathrm{L} ;[\mathrm{MMA}]=9.340$ $\mathrm{mol} / \mathrm{L}$.

A significant induction period was noticed for the AIBN initiated MMA polymerization in the presence of additives $A$ and $B$ ( Fig. 4); a solubility problem prevents the study of other additives in this case. $R_{p}$ was, however, not changed appreciably in presence of the additives (Table II). Interestingly, additives did not impart such noticeable changes during styrene polymerization.

These studies thus indicate that the tetrahedral cobalt complexes (A-D), possessing an unusual coordinately unsaturated nature, selectively bring about the inhibition of radical polymerization of vinyl monomers. In light of literature

TABLE II

$R_{p}$ for Styrene and MMA Polymerization

\begin{tabular}{lccc}
\hline & \multicolumn{3}{c}{$R_{p} \times 10^{4}\left(\mathrm{~mol} \mathrm{~L}^{-1} \mathrm{~s}^{-1}\right)$} \\
\cline { 2 - 4 } Monomer & No additive & $\mathrm{A}^{\mathrm{c}}$ & $\mathbf{B}^{\mathrm{c}}$ \\
\hline Styrene $^{\mathrm{a}}$ & $2.01 \pm 0.02$ & $1.90 \pm 0.02$ & $1.96 \pm 0.02$ \\
MMA $^{\mathrm{b}}$ & $5.86 \pm 0.05$ & $6.82 \pm 0.05$ & $6.60 \pm 0.05$ \\
\hline
\end{tabular}

${ }^{8}[$ styrene $]=8.740 \mathrm{~mol} \mathrm{~L}{ }^{-1} ;\left[\right.$ AIBN] $=0.024 \mathrm{~mol} \mathrm{~L}^{-1}$.

${ }^{b}[\mathrm{MMA}]=9.340 \mathrm{~mol} \mathrm{~L}^{-1} ;\left[\right.$ AIBN] $=0.013 \mathrm{~mol} \mathrm{~L}^{-1}$.

${ }^{c}[A]=[B]=0.009 \mathrm{~mol} \mathrm{~L}^{-1}$. 
reports ${ }^{11}$ regarding the alkyl radical trapping by the cobalt complexes, we believe that present results may stem from a similar radical trapping reaction of the complexes A-D. Further, the "selectivity" in imparting the induction period during polymerization of monomers containing electron withdrawing substituents (MMA and acrylamide) may be a result of the facile redox reaction of these radicals with $\mathrm{Co}$ (II) ion; the electron withdrawing substituents favoring the oxidation of $\mathrm{Co}$ (II) to Co(III). A similar argument was advanced by Lee and Minoura ${ }^{14}$ to explain the selective inhibition observed in the presence of Cr complexes during vinyl polymerization.

For a similar reason, the reaction of the complexes A-D with primary radicals possessing strongly electron withdrawing cyano group is also possible. To corroborate this hypothesis, we have studied the decomposition of AIBN in the presence of complexes A and B (Fig. 5). When AIBN alone is heated in nitrogen atmosphere, it decomposes exothermically with the inception and peak temperatures at 75 and $100^{\circ} \mathrm{C}$, respectively. In the presence of complexes $\mathrm{A}$ and $\mathrm{B}$, the inception temperature shifts to 80 and $90^{\circ} \mathrm{C}$, respectively; the peak temperature remaining unchanged. The area under the exotherm is found to increase significantly in the presence of the complexes. This could be attributed to the reaction of these complexes with the radicals generated during the decomposition of AIBN. The exact structure of the product of such a reaction is not known at the present stage. However, it unambiguously confirms the interaction of these complexes with radicals generated from AIBN decomposition.

We have also observed an order of reaction of 1.5 with respect to MMA in the presence of additives $\mathrm{A}$ and $\mathrm{B}$; the reasons for this deviation from normal kinetic behavior are not known at present.

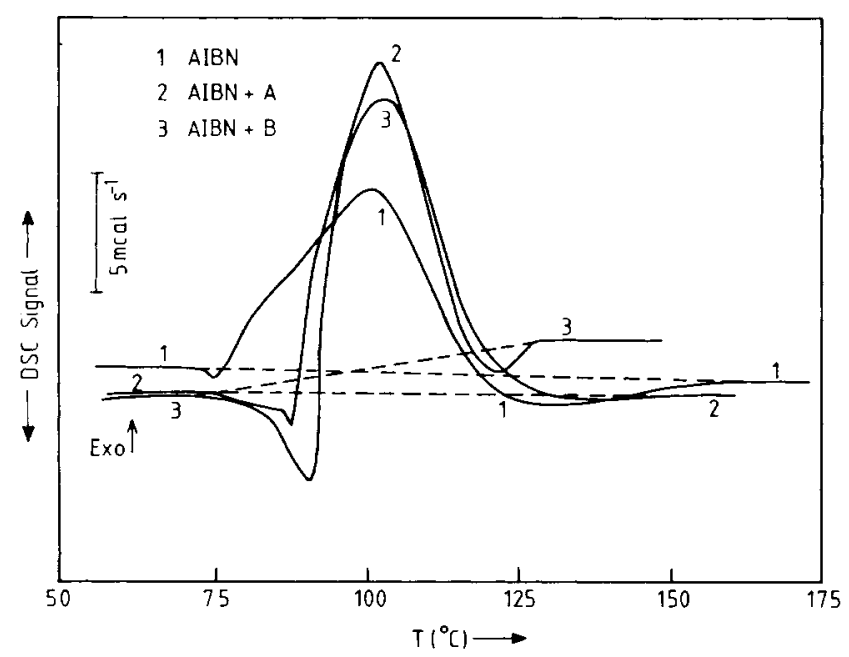

Fig. 5. DSC thermograms for AIBN decomposition. Conditions: nitrogen atmosphere; heating rate: $10^{\circ} \mathrm{C} / \mathrm{min}$; chart speed: $0.5 \mathrm{~min} / \mathrm{cm}$; sensitivity: $50 \mathrm{mV} / \mathrm{cm}$. Sample weight: 1 (AIBN: $3 \mathrm{mg}$ ), 2-3 (AIBN: $3 \mathrm{mg}+$ additive: $3 \mathrm{mg}$ ).

\section{References}

1. S. Tazuke, Prog. Polym. Sci. Jpn., 1, 69 (1971).

2. V. A. Kabanov, J. Polym. Sci. Polym. Symp., 67, 17 (1978). 
3. S. D. Baruah and N. N. Dass, J. Polym. Sci. Polym. Chem. Ed., 19, 513 (1981), and references cited therein.

4. R. C. Samal, J. Macromol. Sci. Chem., A18, 719 (1982).

5. N. S. Yenikolopyan, B. R. Smirnov, G. V. Pomomarev, and I. M. Beigovskii, J. Polym. Sci. Polym. Chem. Ed., 19, 579 (1981).

6. L. R. Melby, A. H. Janowicz, and S. D. Ittel, U.S. Pat. 707,367 (1985); Chem. Abstr., 101, 67837d (1984).

7. H. Hirai and M. Komiyama, J. Polym. Sci. Polym. Chem. Ed., 13, 2419 (1975), and references cited therein.

8. R. D. W. Kemmitt and D. R. Russell, in Comprehensive Organometallic Chemistry, Vol. 5, G. Wilkinson and F. G. A. Stone, Eds., Pergamon, New York, 1982.

9. B. P. Branchand, M. S. Meier, and M. N. Malekzadch, J. Org. Chem., 52, 212 (1987); V. F. Patel and G. Pattenden, J. Chem. Soc. Chem. Commun., 871 (1987).

10. J. Halpern, Science, 227, 869 (1985).

11. L. Sacconi, M. Ciampolini, F. Muggio, and F. F. Cavassion, J. Am. Chem. Soc., 84, 3246 (1962).

12. V. A. Bhanu and K. Kishore, Macromolecules, 22, 3491 (1989).

13. K. Kishore and K. N. Santhanalakshmi, J. Macromol. Sci. Chem., A16, 941 (1981).

14. M. Lee and Y. Minoura, Trans. Faraday Soc., 1649 (1975).

Received June 12, 1989

Accepted March 7, 1990 